\title{
Validação laboratorial de um método automatizado de dosagem da atividade de adenosina desaminase em líquido pleural e em líquido cefalorraquidiano*
}

\author{
Laboratorial validation of an automated assay for the determination \\ of adenosine deaminase activity in pleural fluid and cerebrospinal fluid \\ Márcia Cristina Feres', Maria Cristina De Martino², Suely Maldijian ${ }^{3}$, \\ Fernando Batista ${ }^{4}$, Alexandre Gabriel Júnior ${ }^{5}$, Sérgio Tufik ${ }^{6}$
}

\begin{abstract}
Resumo
Objetivo: A incidência global de tuberculose reforça a necessidade de melhores ensaios para o diagnóstico desta doença, principalmente da tuberculose extrapulmonar. 0 objetivo do trabalho foi validar o desempenho de um método automatizado para a determinação da atividade de adenosina desaminase (ADA) no líquido pleural (LP) e no líquido cefalorraquidiano (LCR), comparando-o com um método convencional (Giusti modificado). Métodos: Selecionaram-se 134 amostras da rotina laboratorial: 94 de LP e 40 de LCR. Foram realizadas as determinações da atividade de ADA através dos dois métodos. Calculou-se a precisão inter- e intra-ensaios, análise de regressão linear, testes de concordância simples e médias das diferenças. Resultados: Os coeficientes de correlação para as amostras de LP e LCR foram, respectivamente, 0,96 e 0,95. A precisão interensaio foi determinada pela média de 21 amostras replicadas em ensaios diferentes para 3 níveis de atividade: baixa, média e alta. Os coeficientes de variação em porcentagem (\%CV) foram, respectivamente, 5,9, 8,1 e 5,8 para amostras de LP; e 21,9, 18,6 e 13,8 para amostras de LCR, respectivamente. A precisão intra-ensaio em \%CV foi, respectivamente, 1,3 e 11,7\% para amostras de LP e LCR. A concordância entre os dois métodos em amostras de LP e LCR foi, respectivamente, 96,8\% e 100\%, considerando-se como valores de referência para o diagnóstico de TB $40 \mathrm{U} / \mathrm{L}$ (convencional) e $30 \mathrm{U} / \mathrm{L}$ (automatizado) em amostras de LP, e $9 \mathrm{U} / \mathrm{L}$ em amostras de LCR para os dois métodos. Conclusões: Os resultados validaram o método automatizado de determinação da atividade de ADA para o uso em amostras de LP e LCR como alternativa ao método convencional.
\end{abstract}

Descritores: Adenosina desaminase; Tuberculose/diagnóstico; Derrame pleural; Líquido cefalorraquidiano.

\begin{abstract}
Objective: The incidence of tuberculosis worldwide has emphasized the need for better assays designed to diagnose the disease, principally the extrapulmonary form. The objective of the present study was to validate the performance of an automated method for the determination of adenosine deaminase (ADA) activity in pleural fluid (PF) and cerebrospinal fluid (CSF), comparing it with a conventional method (the modified Giusti method). Methods: In total, 134 samples were selected from among those tested in our laboratory: 94 PF samples and 40 CSF samples. The ADA activity was determined using the two methods. Inter- and intra-assay precision was determined, linear regression analysis was performed, simple concordance tests were conducted, and the means of the differences were calculated. Results: The correlation coefficients for PF and CSF samples were, respectively, 0.96 and 0.95. Inter-assay precision was determined using 21 replicates at 3 different activity levels: low, medium and high. The percentage coefficient of variation (\%CV) was, respectively, 5.9, 8.1 and 5.8 for PF samples, compared with 21.9, 18.6 and 13.8 for CSF samples. Intra-assay precision in \%CV was 1.3 and 11.7, respectively, for PF and CSF samples. The concordance between the methods in PF and CRF samples was, respectively, $96.8 \%$ and 100\%, considering the reference values for the diagnosis of TB to be $40 \mathrm{U} / \mathrm{L}$ (conventional) and $30 \mathrm{U} / \mathrm{L}$ (automated) in PF samples, versus $9 \mathrm{U} / \mathrm{L}$ (for both methods) in CSF samples. Conclusions: The results validate the use of the automated method of determining ADA activity in PF and CSF samples as an alternative to the conventional method.
\end{abstract}

Keywords: Adenosine deaminase; Tuberculosis/diagnosis; Pleural effusion; Cerebrospinal fluid.

* Trabalho realizado no Departamento de Psicobiologia da Universidade Federal de São Paulo - UNIFESP - e no Laboratório da Associação Fundo de lncentivo à Psicofarmacologia/Medicina Laboratorial - AFIP/ML - São Paulo (SP) Brasil.

1. Biomédica pesquisadora. Departamento de Psicobiologia. Universidade Federal de São Paulo - UNIFESP - São Paulo (SP) Brasil.

2. Coordenadora da Qualidade/Validação e Pesquisa. Laboratório da Associação Fundo de Incentivo à Psicofarmacologia/Medicina Laboratorial - AFIP/ML São Paulo (SP) Brasil.

3. Biomédica. Laboratório Central. Hospital São Paulo/Universidade Federal de São Paulo - HSP/UNIFESP - São Paulo (SP) Brasil.

4. Biomédico. Setor de Bioquímica. Laboratório da Associação Fundo de Incentivo à Psicofarmacologia/Medicina Laboratorial - AFIP/ML - São Paulo (SP) Brasil.

5. Diretor Clínico. Laboratório da Associação Fundo de Incentivo à Psicofarmacologia/Medicina Laboratorial - AFIP/ML - São Paulo (SP) Brasil.

6. Professor Titular do Departamento de Psicobiologia. Universidade Federal de São Paulo - UNIFESP - São Paulo (SP) Brasil.

Endereço para correspondência: Márcia Cristina Feres. Departamento de Psicobiologia, Universidade Federal de São Paulo, Rua Napoleão de Barros, 925, $1^{\circ}$ andar (Secretaria), Vila Clementino, CEP 04024-002, São Paulo, SP, Brasil.

Tel 5511 2149-0155. E-mail: marciaferes@psicobio.epm.br

Apoio financeiro: Este estudo recebeu apoio financeiro do Laboratório da Associação Fundo de Incentivo à Psicofarmacologia/Medicina Laboratorial (AFIP/ML) e da empresa representante Genbiotech Diagnóstica Ltda com doações dos kits e reagentes.

Recebido para publicação em 18/12/2007. Aprovado, após revisão, em 5/5/2008. 


\section{Introdução}

A incidência da tuberculose (TB) no mundo tem aumentado; estimam-se 100.000 novos casos a cada ano e $80 \%$ destes estão concentrados em um grupo de 22 países, no qual o Brasil está incluído.(1,2) Nos países ocidentais, verifica-se um aumento de casos de TB pulmonar e extrapulmonar, principalmente devido à prevalência de infecção pelo HIV. ${ }^{(3)}$

0 laboratório clínico reúne uma série de testes que contribuem para o diagnóstico da TB. Podemos citar como prioritária a pesquisa de micobactérias através do teste direto pela coloração de Ziehl-Neelsen. Este é extremamente importante, pois permite o início imediato do tratamento quando positivo. Além disso, tem baixo custo e é de fácil e rápida execução. Paralelamente, realiza-se a cultura em meio de Löwenstein-Jensen ou similar. Entretanto, o principal problema da baciloscopia é a sua baixa sensibilidade. ${ }^{(2,4)} A$ cultura, embora mais sensível, tem como fator limitante o resultado tardio, comprometendo sobremaneira o tratamento. (3) No caso de líquido pleural (LP), o rendimento dos testes citados é próximo de zero para a baciloscopia, enquanto que a cultura varia de $10 \%$ a $35 \%$, o que dificulta ainda mais o diagnóstico. ${ }^{(5)}$

Os métodos de detecção automatizada ou semi-automatizada do crescimento micobacteriano em meios líquidos possibilitam o diagnóstico mais precoce, com a detecção entre uma e três semanas, ao invés das três a oito semanas com o uso do meio sólido. Dentre estes métodos, podemos citar o sistema de detecção radiométrico rápido de micobactérias em espécime clínico BACTEC 460 TB R (Becton Dickinson, Sparks, MD, EUA), o qual está bem estabelecido, com validação internacional e aprovado pela Organização Mundial da Saúde. ${ }^{(6)}$ Entretanto, trabalhos apontam uma taxa de culturas falso-positivas entre 1,4\% e 4\%, devido à contaminação entre os espécimes durante a leitura pelo equipamento, além do problema gerado pelo descarte de material radioativo. ${ }^{(6)}$

No Brasil, este sistema está entrando em desuso e sendo substituído por outros ditos mais rápidos e não radiométricos em laboratórios de referência, após sua avaliação quanto à relação de custo-efetividade em diferentes situações. ${ }^{(7)}$

A biópsia auxilia no diagnóstico diferencial entre o granuloma com necrose caseosa e outras lesões granulomatosas inespecíficas sem necrose, princi- palmente nos casos em que o estudo microbiológico não foi suficiente para estabelecer o diagnóstico etiológico. ${ }^{(5,6)}$

Até o presente momento, os testes sorológicos que realizam pesquisa de anticorpos empregando as metodologias de enzimaimunoensaio e radioimunoensaio não são tão definitivos como a demonstração do agente etiológico propriamente dito, uma vez que esses ensaios freqüentemente apresentam especificidade reduzida..$^{(5,7)}$

0 teste intradérmico, baseado na reação de hipersensibilidade tardia após o contato com o derivado protéico purificado, pode ser usado apenas como triagem, pois o resultado positivo indica exposição ao bacilo ou à cepa vacinal. A vacinação em massa no Brasil, com bacilo de Calmette-Guérin, é um fator limitante para utilização desta prova. ${ }^{(8)}$ Ressalta-se que pacientes imunodeprimidos apresentam reação negativa mesmo na presença de cultura positiva ou infecção ativa. ${ }^{(2,9)}$

A detecção do ácido tuberculoesteárico presente no Mycobacterium tuberculosis pode ser realizada por cromatografia gasosa e espectrometria de massa. Apesar de mais sensíveis, estas técnicas são menos específicas, bem mais complexas e onerosas.(1)

Se para o diagnóstico de TB pulmonar ainda temos dificuldades para encontrar metodologias que atendam as necessidades da prática clínica, no caso da TB extrapulmonar a situação torna-se um problema ainda maior. Os métodos convencionais, úteis para o diagnóstico de TB pulmonar, têm baixo desempenho quando realizados no LP ou em outros líquidos cavitários nos casos de TB extrapulmonar. ${ }^{(1,7)}$

0 reconhecimento destas dificuldades no diagnóstico da TB levou a busca de outros métodos que pudessem otimizar a abordagem do paciente com derrame pleural e suspeita dessa doença nas diversas formas extrapulmonares, tais como meníngea, renal, pleural, óssea e ganglionar. Dentre as novas técnicas, destacam-se a reação em cadeia da poli-

Tabela 1 - Precisão intra-ensaio determinada pela média, desvio-padrão e coeficiente de variação das repetições.

\begin{tabular}{lrcc}
\hline \multicolumn{1}{c}{ Tipo de amostra } & $\begin{array}{c}\text { Média, } \\
\mathrm{U} / \mathrm{L}\end{array}$ & $\mathrm{dp}, \mathrm{U} / \mathrm{L}$ & $\mathrm{CV}, \%$ \\
\hline Líquido pleural & 16,3 & 0,20 & 1,3 \\
Líquido cefalorraquidiano & 0,6 & 0,07 & 11,7 \\
\hline
\end{tabular}

CV: coeficiente de variação. CV $=1,3 \pm 0,2 \%$ para líquido pleural; e $\mathrm{CV}=11,6 \pm 0,07 \%$ para líquido cefalorraquidiano. 
merase, que identifica o DNA do $M$. tuberculosis por amplificação de segmentos conhecidos do seu genoma, e as que detectam marcadores bioquímicos produzidos durante o processo inflamatório, desencadeado pelo $M$. tuberculosis, tais como interferon gama e a adenosina desaminase (ADA). ${ }^{(4,10)}$

Esta denominação-ADA-é uma generalização dada a um grupo de enzimas com pesos moleculares distintos que possuem uma função química semelhante no metabolismo das purinas, catalisando a conversão da adenosina e da desoxiadenosina em inosina e desoxiinosina, respectivamente. ${ }^{(9)}$ A ADA está presente em quase todos os vertebrados; no homem, é encontrada principalmente nos linfócitos T e está diretamente relacionada à ativação dessas células. A ativação dos linfócitos T pode ocorrer em várias situações clínicas onde encontramos níveis elevados de ADA. A sua quantificação é útil particularmente nos casos de meningites e pleurites tuberculosas. ${ }^{(3,10-12)}$ Desta forma, a determinação de ADA tem uma forte relevância para o diagnóstico laboratorial no contexto clínico do paciente. ${ }^{(12)}$

Pesquisadores observaram que a atividade de ADA no soro está aumentada em pacientes com hepatites agudas e cirroses hepáticas, dentre outras afecções. ${ }^{(10)}$ Outros autores fizeram estudos relacionando níveis de atividade de ADA com algumas doenças, tais como artrite reumatóide, doenças linfoproliferativas, lúpus eritematoso sistêmico, psoríase e eclampsia. ${ }^{(13-15)}$ No entanto, no soro, a atividade de $A D A$ tem sido utilizada muito mais para o seguimento da TB do que para o seu diagnóstico, haja vista a sua inespecificidade. Na literatura, há inúmeros trabalhos que envolvem a dosagem de $\mathrm{ADA}$ em casos com suspeita de $\mathrm{TB}$, principalmente a extrapulmonar, e que consideram este grupo de enzimas como um marcador bioquímico valioso para esta doença. ${ }^{(7,9,10)}$

Esta determinação tem sido realizada através do método proposto por Giusti em 1974 ${ }^{(16,17)}$ e que sofreu algumas modificações no decorrer dos anos, acrescentando-se a reação de Berthelot ${ }^{(3,10,16)}$ para a obtenção de melhores resultados (Giusti modificado). Embora considerada eficaz, esta metodologia ainda possui uma série de limitações: necessita de preparação de reagentes, tem execução manual, com leitura em espectrofotômetro, sendo que todos estes passos são desenvolvidos in house, ou seja, por cada laboratório individualmente, não existindo, portanto, uma padronização que permita fazer
Tabela 2 - Precisão interensaio em três níveis diferentes (I, II e III) utilizando amostras de líquido pleural e de líquido cefalorraquidiano.

\begin{tabular}{cccc}
\hline Nível & Média, U/L & dp, U/L & CV, \% \\
\hline $\mathrm{I}^{\mathrm{a}}$ & 5,9 & 0,4 & 5,9 \\
$\mathrm{II}^{\mathrm{a}}$ & 19,4 & 1,6 & 8,1 \\
III $^{\mathrm{a}}$ & 60,1 & 3,5 & 5,8 \\
$\mathrm{I}^{\mathrm{b}}$ & 0,8 & 0,2 & 21,9 \\
II $^{\mathrm{b}}$ & 5,4 & 1,0 & 18,6 \\
III $^{\mathrm{b}}$ & 7,2 & 1,0 & 13,8 \\
\hline
\end{tabular}

${ }^{\mathrm{a} A m o s t r a s ~ d e ~ l i ́ q u i d o ~ p l e u r a l ; ~ e ~}{ }^{\mathrm{b}}$ amostras de líquido cefalorraquidiano.

deste ensaio um parâmetro que preencha todos os critérios de um bom teste diagnóstico. ${ }^{(18)}$

0 objetivo do presente trabalho foi testar um método automatizado com um kit comercial de determinação da atividade de ADA no soro, padronizando-o pela primeira vez para amostras de LP e de líquido cefalorraquidiano (LCR), comparando os resultados com o método de Giusti modificado, tido como teste de referência para o nosso estudo bioquímico. $^{(16)}$

\section{Métodos}

Foram selecionadas da rotina do Laboratório Central do Hospital São Paulo/Universidade Federal de São Paulo (HSP/UNIFESP) e da rotina do Laboratório da Associação Fundo de Incentivo à Psicofarmacologia/Medicina Laboratorial 94 amostras de LP e 40 amostras de LCR com valores de atividade de ADA previamente determinados pelo método convencional-Giusti modificado, in housetotalizando 134 amostras estudadas.

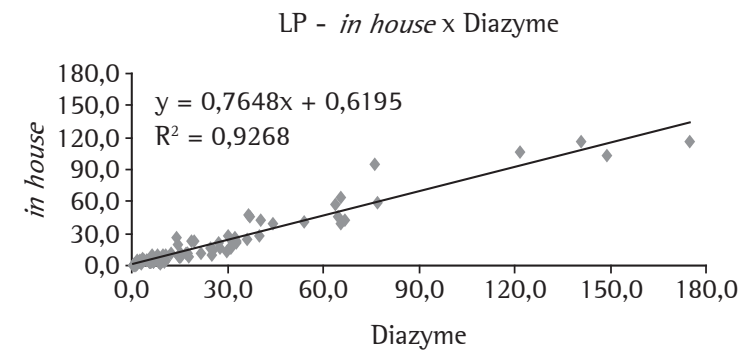

Figura 1 - Gráfico da correlação, através do teste de regressão linear, entre os métodos convencional (in house) e automatizado (Diazyme) para amostras de líquido pleural (LP). 0 índice de correlação (r) foi de 0,96. 


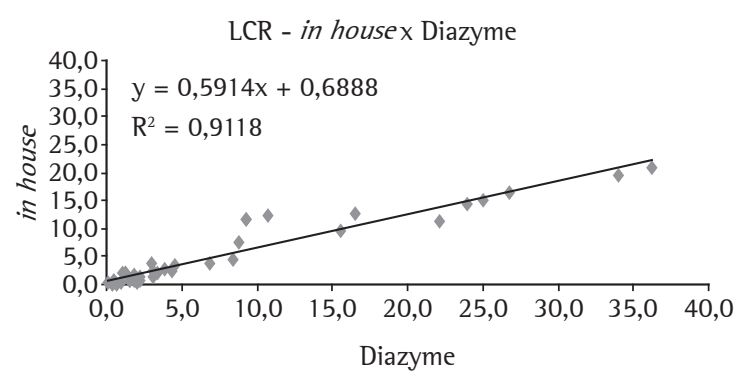

Figura 2 - Gráfico da correlação, através do teste de regressão linear, entre os métodos convencional (in house) e automatizado (Diazyme) para amostras de líquido cefalorraquidiano (LCR). 0 índice de correlação (r) foi de 0,95 .

Realizaram-se nestas amostras as repetições das dosagens de ADA pelo método de Giusti modificado e simultaneamente pelo método com o kit comercial Diazyme (Diazyme Laboratories, San Diego, CA, EUA) com automação em equipamento ADVIA 1650 (Bayer Diagnostics, Tarrytown, NY, EUA).

Para a realização do método de Giusti modificado, ${ }^{(16)}$ após a centrifugação das amostras, $25 \mu \mathrm{L}$ dos sobrenadantes foram transferidos para um tubo de ensaio e $500 \mu \mathrm{L}$ de uma solução de adenosina foram adicionados. A mistura foi aquecida por $60 \mathrm{~min}$ a $37^{\circ} \mathrm{C}$, e a reação foi interrompida com adição de uma solução de fenol e nitroprussiato e uma solução de hipoclorito. Em seguida, a solução resultante foi aquecida por $30 \mathrm{~min}$ a $37^{\circ} \mathrm{C}$. A leitura do índice de amônia liberada pela ação da ADA foi feita em espectrofotômetro a $620 \mathrm{~nm}$. As mesmas amostras foram submetidas à dosagem de ADA pelo método de Diazyme automatizado, utilizando-se, para a realização do teste, $11 \mu \mathrm{L}$ de amostra de LP e $11 \mu \mathrm{L}$ de controle comercial em dois níveis de atividade (alto e baixo). Em amostras de $\mathrm{LCR}$, foram utilizados $22 \mu \mathrm{L}$. Os resultados dos dois métodos foram expressos em U/L.

Para o método automatizado, foram realizados testes de precisão inter- e intra-ensaios. A precisão intra-ensaio foi realizada com 21 amostras replicadas de $\mathrm{LP}$ e LCR. A precisão interensaio foi determinada em 21 ensaios separados, sendo que essa precisão foi determinada utilizando um pool de LP e LCR em três níveis diferentes de valores de atividade de ADA. Para os dois tipos de precisão, foram calculados a média, desvio-padrão e coeficiente de variação (CV) (Tabelas 1 e 2).

Os resultados do método convencional e do método automatizado foram submetidos aos testes de correlação (Figuras 1 e 2 ) e de concordância simples para atestar a equivalência entre eles, ${ }^{(17-19)}$ considerando-se, para a confirmação de TB em amostras de $\mathrm{LP}$, o valor de corte de $40 \mathrm{U} / \mathrm{L}$ para o método de Giusti modificado e de $30 \mathrm{U} / \mathrm{L}$ para o método automatizado. Para a confirmação de TB em amostras de LCR, considerou-se o valor de corte de $9 \mathrm{U} / \mathrm{L}$ para ambos os métodos (Tabela 3).

Durante o estudo, avaliou-se a estabilidade dos reagentes do método automatizado por um período de um ano. Também foi avaliado o volume de amostra necessário, tempo de execução e liberação dos resultados com a realização dos ensaios por esse método.

\section{Resultados}

A Tabela 1 mostra a precisão intra-ensaio para cada tipo de líquido biológico, determinada a partir da média de 21 amostras replicadas ensaiadas, tanto de LP quanto de LCR.

Tabela 3 - Teste de concordância simples dos resultados obtidos nos métodos convencional e automático para amostras de líquido pleural e de líquido cefalorraquidiano.

\begin{tabular}{cccc}
\hline $\begin{array}{c}\text { Método automatizado: } \\
\text { líquido pleural }\end{array}$ & $\begin{array}{c}\text { Método convencional } \\
\text { superior a } 40 \mathrm{U} / \mathrm{L}\end{array}$ & $\begin{array}{c}\text { Método convencional - } \\
\text { inferior a 40 U/L }\end{array}$ & Total \\
\hline Superior a 30 U/L & 14 & 2 & 16 \\
Inferior a 30 U/L & 1 & 77 & 78 \\
Total & 15 & 79 & $94^{*}$ \\
Método automatizado: & Método convencional - & Método convencional - & Total \\
líquido cefalorraquidiano & superior a 9 U/L & 0 & 11 \\
Superior a 9 U/L & 11 & 29 & 29 \\
Inferior a 9 U/L & 0 & 29 & $40^{* *}$ \\
Total & 11 & $29 / \mathrm{L}$ & \\
\hline
\end{tabular}

*Concordância de 96,8\%; e *** concordância de 100\%. 
A Tabela 2 mostra a precisão interensaio determinada a partir da média de duplicatas em 21 ensaios separados, para três níveis diferentes de atividade (I, II e III) de ADA nas amostras de LP e LCR. Foram calculados a média, desvio-padrão e CV.

Para as amostras de $\mathrm{LP}$, a precisão interensaio nos três níveis de atividade de ADA, os valores de $\%$ CV obtidos não ultrapassaram 10\%, enquanto que, para as amostras de LCR, os valores de \%CV variaram de $13 \%$ a $20 \%$ em média.

As Figuras 1 e 2 mostram os gráficos de correlação entre os dois métodos para amostras de LP e de LCR com diferentes níveis de atividade.

A Tabela 3 apresenta os valores do teste de concordância simples considerando os valores de corte de $40 \mathrm{U} / \mathrm{L}$ para o método convencional e de $30 \mathrm{U} / \mathrm{L}$ para o método automatizado para detecção de TB pleural, com concordância de $96,8 \%$ nas amostras de LP. Com o valor de corte para TB meníngea de $9 \mathrm{U} / \mathrm{L}$ nas amostras de LCR para os dois métodos, encontrou-se 100\% de concordância.

\section{Discussão}

A avaliação da eficiência de testes diagnósticos alternativos para identificação da TB é de grande relevância, em particular a quantificação da atividade de ADA, que pode ser empregada em países cuja casuística de TB é de grande prevalência, como a Organização Pan-Americana de Saúde anuncia. Além disso, ela pode, em muitos casos, favorecer a confirmação diagnóstica substituindo a biópsia, laparoscopia e outros exames que fazem a comprovação definitiva do diagnóstico, mas que são mais sofisticados, caros e indisponíveis em muitos serviços de saúde.

As sociedades de pneumologia, incluindo a Sociedade Brasileira de Pneumologia e Tisiologia $(\mathrm{SBPT}),{ }^{(20)}$ recomendam a determinação da atividade de ADA em líquidos orgânicos, principalmente em LP e LCR. ${ }^{(21)}$ Portanto, a padronização de métodos automatizados confiáveis se faz necessária para definir diagnósticos de maneira mais rápida e favorecer o tratamento da TB.

No entanto, a praticidade e a rapidez do método automatizado proposto neste trabalho devem ser acompanhadas por uma validação adequada e útil para que o método possa ser utilizado em larga escala. ${ }^{(22)}$ Há um grande interesse laboratorial e clínico em padronizar um método automatizado substituindo o uso de metodologias in house, como o método de Giusti modificado por Berthelot, que, apesar de ser considerado muito indicado e estar disponível há pelo menos duas décadas, sofre influência de diversos fatores de interferência relacionados a execução manual da técnica, diminuindo sua precisão, exatidão e, conseqüentemente, sua correlação inter- e intra-laboratorial. Além disso, a preparação dos reagentes específicos de alta toxidade para uso no teste demanda profissionais preparados e qualificados.

As amostras de líquidos orgânicos (LP e LCR) foram selecionadas aleatoriamente, oriundas de pacientes internados no HSP/UNIFESP e portadores de várias doenças, dentre elas as infecciosas, imunológicas e neoplásicas. Neste trabalho, não se enfocou a clínica, pois o interesse dos autores se voltou mais para a validação da metodologia laboratorial de ADA e, portanto, as amostras selecionadas possuíam valores de atividade deste grupo de enzimas que se enquadravam numa grande gama de variações (valores altos, médios e baixos).

Os índices encontrados nos dois tipos de líquidos orgânicos confirmaram a forte correlação entre os dois métodos (Figuras 1 e 2).

A importância da dosagem de ADA em líquidos orgânicos já é bem estabelecida, ${ }^{(11,23,24)}$ mas o nível de corte ideal para este teste, no diagnóstico da TB, ainda permanece discutível.

No presente trabalho, para amostras de LP, considerou-se o nível de corte de $40 \mathrm{U} / \mathrm{L}$ para o método convencional, segundo recomendações da SBPT, ${ }^{(8,12,19)}$ embora vários trabalhos associem o aspecto clínico a diferentes pontos de cortes para ADA. ${ }^{(21)}$ Para o método automatizado, sugerimos um valor de corte de $30 \mathrm{U} / \mathrm{L}$ após o cálculo da média percentual das diferenças de resultados entre os dois métodos, pelo qual se encontrou um valor em torno de 30\% menor para o automatizado. Com estes níveis de corte, o teste de concordância simples apresentou 96,8\% de concordância entre os métodos, de acordo com estudos da literatura. ${ }^{(23)}$

Para amostras de LCR, o valor de corte preconizado pela literatura é de $9 \mathrm{U} / \mathrm{L}$ para o diagnóstico da TB. Considerou-se este valor para os dois métodos, pois utilizando-se este valor obteve-se 100\% de concordância no teste de concordância simples, embora a média percentual das diferenças dos resultados entre os dois métodos foi em torno de $35 \%$. Provavelmente este valor poderá ser mais 
detalhadamente analisado e reavaliado em trabalhos subseqüentes, selecionando-se amostras de pacientes com diagnóstico definido.

Os testes de precisão inter- e intra-ensaios foram satisfatórios com \%CV adequados para as amostras de LP. No entanto, para as amostras de LCR, a \%CV foi mais alta porque os níveis de atividade de ADA são menores em LCR do que nos outros líquidos cavitários. Além disso, trata-se de líquido muito menos rico em elementos protéicos e sabidamente apresenta diferenças enzimáticas e imunológicas mais instáveis, haja vista que para os mesmos níveis de atividade de ADA em LP (nível I) encontrou-se \%CV maiores do que em LCR (níveis II e III).

Outros aspectos analisados durante a execução do método automatizado incluem a estabilidade dos reagentes, que permaneceu inalterada durante 6 meses a $4^{\circ} \mathrm{C}$, a rapidez na obtenção dos resultados-em torno de 10 min-e o volume de amostra menor.

Através dos resultados obtidos neste estudo, o método automatizado, realizado como acima descrito, apresentou-se adequado para substituir o método convencional in house sem comprometimento da interpretação do resultado final.

A partir do momento de sua implantação na rotina laboratorial, poderão ser realizados estudos clínicos analisando a sensibilidade e a especificidade ${ }^{(22,25)} \mathrm{do}$ método automatizado para o diagnóstico da TB e de outras enfermidades que estimulam o aumento da atividade de ADA, bem como dispor de métodos padronizados, possibilitando uma melhor comparação de resultados interlaboratoriais.

\section{Agradecimentos}

À Dra. Rosali Teixeira da Costa, chefe do Ambulatório de Pneumologia da UNIFESP os esclarecimentos prestados e a disponibilidade em nos atender.

\section{Referências}

1. Dinnes J, Deeks J, Kunst H, Gibson A, Cummins E, Waugh $\mathrm{N}$, et al. A systematic review of rapid diagnostic tests for the detection of tuberculosis infection. Health Technol Assess. 2007;11(3):1-196.

2. Bem AF, Caputi CS, Meireles FB, Vargas AP, Carlotto MS, Huber IC. Valor diagnóstico da determinação da atividade da adenosina deaminase no derrame pleural tuberculoso em indivíduos infectados e não infectados pelo HIV. Rev Bras Anal Clin. 2005;37(1):23-6.
3. Goto M, Noguchi Y, Koyama H, Hira K, Shimbo T, Fukui $\mathrm{T}$. Diagnostic value of adenosine deaminase in tuberculous pleural effusion: a meta-analysis. Ann Clin Biochem. 2003;40(Pt 4):374-81.

4. Seiscento M, Conde MB, Dalcomo MM. Tuberculous pleural effusions. J Bras Pneumol. 2006;32(4):S174-81

5. Neves DD, Dias RM, Cunha AJ, Chibante AM. Rendimento de variáveis clínicas, radiológicas e laboratoriais para o diagnóstico da tuberculose pleural. J Pneumol. 2004;30(4):409-16.

6. Somoskövi A, Ködmön C, Lantos A, Bártfai Z, Tamási L, Füzy J, et al. Comparison of recoveries of mycobacterium tuberculosis using the automated BACTEC MGIT 960 system, the BACTEC 460 TB system, and Löwenstein-Jensen medium. J Clin Microbiol. 2000;38(6):2395-7.

7. Kritski AL, Conde MB, Souza GR. Exames complementares utilizados no diagnóstico da tuberculose. In: Kritski AL, Conde MB, Souza GR, editors. Tuberculose: Do ambulatório à enfermaria. 1ª ed. São Paulo: Ed.Ateneu; 2000. p. 54.

8. Viamont A. Diagnóstico de meningite tuberculosa através de enzimaimunoensaio (ELISA), utilizando PPD como antígeno [dissertation]. São Paulo (SP): Universidade Federal de São Paulo; 1992.

9. Caldas AC, Neves DD, Dias RM. Rendimento da dosagem da adenosina deaminase para o diagnóstico da tuberculose pleural em região de alta prevalência da doença. Pulmão RJ. 2004;13(1):19-23.

10. Greco S, Girardi E, Masciangelo R, Capoccetta GB, Saltini C. Adenosine deaminase and interferon gamma measurements for the diagnosis of tuberculous pleurisy: a meta-analysis. Int J Tuberc Lung Dis. 2003;7(8):777-86.

11. Lamsal M, Gautam N, Bhatta N, Majhi S, Baral N, Bhattacharya SK. Diagnostic utility of adenosine deaminase (ADA) activity in pleural fluid and serum of tuberculous and non-tuberculous respiratory disease patients. Southeast Asian J Trop Med Public Health. 2007;38(2):363-9.

12. Kashyap RS, Ramteke SP, Deshpande PS, Purohit HJ, Taori GM, Daginawala HF. Comparison of an adenosine deaminase assay with ELISA for the diagnosis of tuberculous meningitis infection. Med Sci Monit. 2007;13(9):BR200-204.

13. Lima 1, Néri F, Barreto S, Mittermayer BS. Dosagem sérica de adenosina deaminase em lúpus eritematoso sistêmico: ausência de associação com atividade de doença. Rev Bras Reumatol. 2005:45(5):273-79.

14. Erbagci Z, Erbagci AB, Köylüoglu O, Tuncel AA. Serum adenosine deaminase activity in monitoring disease activity and response to therapy in severe psoriasis. Acta Medica (Hradec Kralove). 2006;49(2):101-4.

15. Kafkasli A, Karabulut AB, Atmaca R, Laurini R. Clinical correlation between adenosine deaminase activity and pre-eclampsia severity. J Int Med Res. 2006;34(3):247-55.

16. Giusti G. Adenosine deaminase. In: Bergmeyer HU, editor. Methods of enzymatic analysis. 2nd edition. New York: Academic Press; 1974. p. 1092-99.

17. Grisi SJ, Cardoso AC, Bellizia L, Escobar AM. Tuberculose peritoneal: relato de caso e comparação de métodos diagnósticos. Pediatria (São Paulo). 2001;23(1):100-5.

18. Passing $\mathrm{H}$, Bablok. A new biometrical procedure for testing the equality of measurements from two different analytical methods. Application of linear regression procedures for method comparison studies in clinical chemistry, Part 1. J Clin Chem Clin Biochem. 1983;21(11):709-20. 
19. Diacon AH, Van de Wal BW, Wyser C, Smedema JP, Bezuidenhout J, Bolliger CT, et al. Diagnostic tools in tuberculous pleurisy: a direct comparative study. Eur Respir J. 2003;22(4):589-91.

20. Sociedade Brasileira de Pneumologia e Tisiologia. 1 Consenso Brasileiro de Tuberculose. J Pneumol. 1997;23(6):279-346.

21. Smach MA, Garouch A, Charfeddine B, Ben Abdelaziz A, Dridi $\mathrm{H}$, Krayem B, et al. Diagnostic value of serum and pleural fluid adenosine deaminase activity in tuberculous pleurisy [Article in French]. Ann Biol Clin (Paris). 2006;64(3):265-70.

22. Ribani M, Bottoli CB, Collins CH, Jardim IC, Melo LF. Validação em métodos cromatográficos e eletroforéticos. Quím Nova. 2004;27(5):771-8.
23. Kaisemann MC, Kritski AL, Pereira MFC, Trajman A. Dosagem da atividade da adenosina deaminase no líquido pleural para o diagnóstico da tuberculose pleural. J Pneumol. 2004;30(6):549-56.

24. Valdés L, San José E, Alvarez D, Valle JM. Adenosine deaminase (ADA) isoenzyme analysis in pleural effusions: diagnostic role, and relevance to the origin of increased ADA in tuberculous pleurisy. Eur Respir J. 1996;9(4):747-51.

25. Comité de Garantía de la Calidad y Acreditación de Laboratorios. Comisión de Calidad Analítica da Sociedad Española de Química Clínica y Patologia Molecular. Proceedings of the Gestion de La Calidad En El Laboratorio Clinico; 2006 Apr 7-8; Santiago, Chile. 